University for Business and Technology in Kosovo

UBT Knowledge Center

UBT International Conference

2014 UBT International Conference

Nov 8th, 11:45 AM - 12:00 PM

\title{
Concept of Online Assisted Platform for Technologies and Management in Communications - OPTIMEK
}

\author{
Galia Marinova \\ Technical University of Sofia, gim@tu-sofia.bg \\ Vassil Guliashki \\ Institute of Information and Communication Technologies, vggul@yahoo.com \\ Ognyan Chikov
}

Follow this and additional works at: https://knowledgecenter.ubt-uni.net/conference

Part of the Computer Sciences Commons

\footnotetext{
Recommended Citation

Marinova, Galia; Guliashki, Vassil; and Chikov, Ognyan, "Concept of Online Assisted Platform for Technologies and Management in Communications - OPTIMEK" (2014). UBT International Conference. 58.

https://knowledgecenter.ubt-uni.net/conference/2014/all-events/58
}

This Event is brought to you for free and open access by the Publication and Journals at UBT Knowledge Center. It has been accepted for inclusion in UBT International Conference by an authorized administrator of UBT Knowledge Center. For more information, please contact knowledge.center@ubt-uni.net. 


\title{
Concept of Online Assisted Platform for Technologies and Management in Communications - OPTIMEK
}

\author{
Galia Marinova $^{1}$, Vassil Guliashki ${ }^{2}$, Ognyan Chikov ${ }^{1}$ \\ ${ }^{1}$ Technical University of Sofia, Faculty of Telecommunications, \\ ${ }^{2}$ Institute of Information and Communication Technologies - BAS, Section Information processes \\ and decision support systems \\ gim@tu-sofia.bg1,vggul@yahoo.com²
}

\begin{abstract}
The paper describes the concept of a Multimodular Multydisciplinary platform, contacting through unified templates in a Portal with knowledge, with Useful INTERNET resources, in order to provide advanced research and education. Usually the online resources available are mainly in the area of e- and distance education, but still an understanding is missing for the scale and the use of studying and the systematization of the online resource. The new concept has an accent of the useful INTERNET resource and the development of a System of nets to it, in the aim of solving tasks and generating new knowledge in the area of communications. It will help advanced and accelerated scientific research. The concept implements three actual trends in scientific research in communications: system approach, optimal use of INTERNET resources and INTERNET exposition, and common scientific research in technologies and management. OPTIMEK is constructed from Modules, which organize Local resources and a Portal with knowledge with Online assistants for technologies and management in communications. Each Module in OPTIMEK includes Local resource and Online assistant. The Local resources in the module consist in a local configuration of hardware equipment and software tools. The Online assistant of the Module consists in, online downloadable tools or calculators and a System of nets. Examples are given for interaction between 2 of the Modules in OPTIMEK for specific problems solving - Module for Computer-aided design in communications and Module for Optimization tasks in communications.
\end{abstract}

Keywords: online assistant, optimek, optimization, communication

\section{Introduction}

The paper describes the concept of a Multimodular Multydisciplinary platform [1,2], contacting through unified templates in a Portal with knowledge, with Useful INTERNET resources, in order to provide advanced research and education.

Usually the online resources available are mainly in the area of e- and distance education, but still an understanding is missing for the scale and the use of studying and systematization of the online resources. The new concept has an accent on the useful INTERNET resources and the development of a System of nets to it, with the aim to solve tasks and to generate new knowledge in the area of communications. It will help advanced and accelerated scientific research.

The concept implements three actual trends in scientific research in communications: system approach, optimal use of INTERNET resources and INTERNET exposition, and common scientific research in technologies and management. OPTIMEK is constructed from Modules, which organize Local resources and a Portal with knowledge with Online assistants for technologies and management in communications. Each Module in OPTIMEK includes Local resource and Online assistant. The Local resources in the module consist in a local configuration of hardware equipment and software tools. The Online assistant of the Module consists in online downloadable tools or calculators, Open source code and a System of nets.

The methods and tools for the software realization of OPTIMEK are described and the implementation of cloud technologies is considered.

Examples are given for interaction between 2 of the Modules in OPTIMEK for specific problems solving - Module for Computer-aided design in communications and Module for Optimization tasks in communications. 


\section{Structure, Modules and Tasks to be solved in OPTIMEK}

\subsection{Structure of OPTIMEK}

The structure of the platform OPTIMEK is presented on Fig. 1. The main elements in OPTIMEK are:

- Portal with knowledge for assisting technologies and management in communications;

- Online assistants to the Modules for technologies and management in communications;

- Local resources in the modules;

- System of nets;

- CAD cloud.
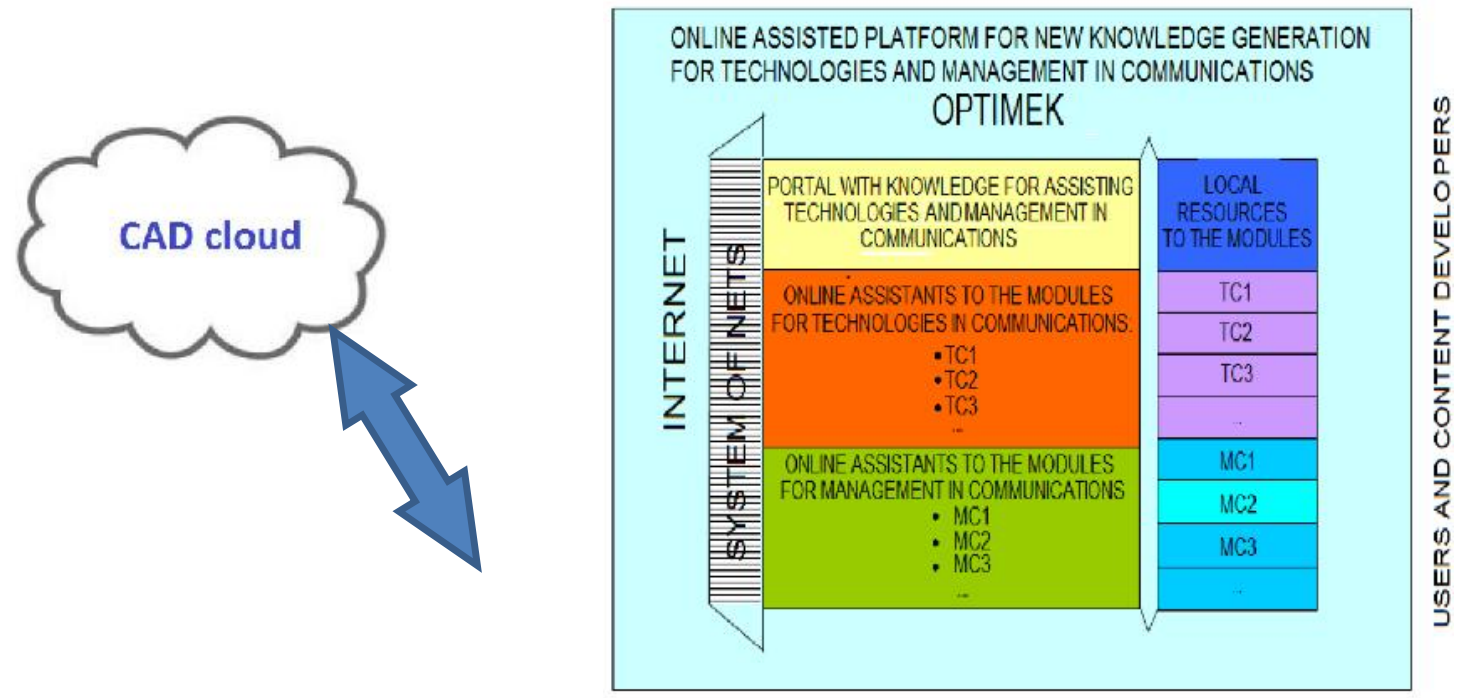

STUDENTS, RESERCHERS, MANAGERS, COMPANIES

Fig. 1. Structure of OPTIMEK - online assistance and cloud technologies

The modules in OPTIMEK, planned to be developed first are:

Five modules in communication technologies:

> Module TC1: Materials and construction in communications,

$>$ Module TC2: Computer-aided design in communications,

$>$ Module TC3: Data storage, security and management,

$>$ Module TC4: Algorithms for software defined radio,

$>$ Module TC5: Optimization tasks in communications.

Three modules in management technologies:

> Module MC1: Norms, legislation, intellectual property and glossary in communications,

$>$ Module MC2: System of barcodes,

$>$ Module MC3: Economic analysis of communication products.

The structure of a module in OPTIMEK is presented on Fig.2. The Concept of the Web assistants combined to Local resources, for CAD of communication circuits and systems is presented on Fig.3. 


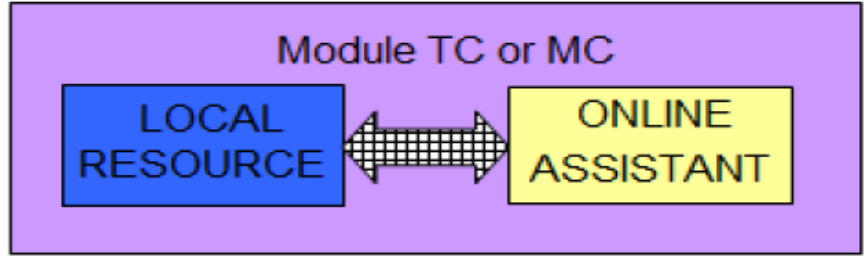

Fig. 2. Structure of a module in OPTIMEK

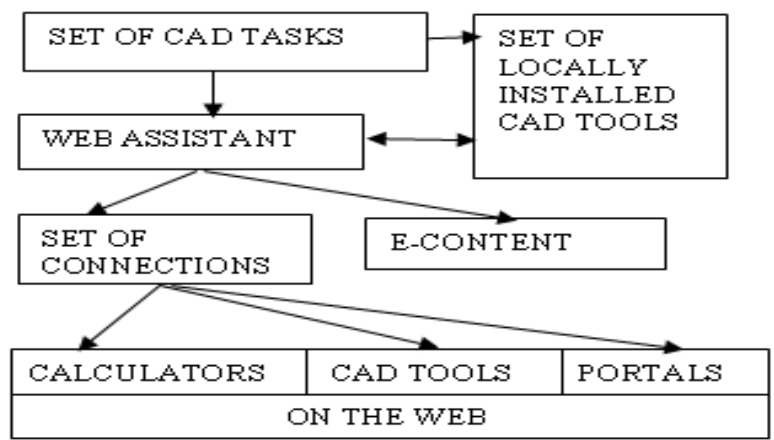

Fig.3. The Concept of the Web assistants combined to Local resources, for CAD of communication circuits and systems

\subsection{Structure of the Module TC2: Computer-aided design in communications and Module TC5: Optimization tasks in communications}

The Online assistant to the module TC2: Computer-aided design in communications consists in:

- Development of a System of nets to the Set of the useful INTERNET resources:

$>$ Connection to a set of online tools and calculators for assisting the computer-aided design of communication circuits: SMPS, nanocommunication elements, PCBs, antennas and others;

$>$ Connection to Open source platforms for computer-aided design in communications.

$>$ Connection to portals of CAD systems users, as YOUSPICE and others.

- Development of a System for project exchange for circuits through the Portal with knowledge and cloud technologies.

- Development of E-Content - Learning modules, Illustrative examples, Guidelines for work.

New tools will be developed and/or integrated, as for example:

$>$ Development of Online tool for modeling and test of pseudorandom number generators PRNGs [6].

$>$ Development of a System of nets to norms for testing PRNGs and for DC/DC converter design [1].

$>$ Online circuit calculators [4].

The module TC5: Optimization tasks in communications integrates the WEB-based System WebOptim $[14,15]$, developed in the Institute for Information and Communication Technologies IICT-BAS. This is a decision support system designed to solve single objective and multiple objective optimization problems in the platform OPTIMEK. It creates resource for optimization problems solving at each stage of the technological design and research, and of the management in the communications.

The local resource for the module TC5 is the following: 
The WEB-based system WebOptim is developed (see: http://weboptim.iinf.bas.bg/). This is a decision support system designed to solve single objective and multiple objective optimization problems, corresponding to a broad class real application problems. The optimization problems in the communications take an important place among them.

The system is developed by means of software technologies, given by Microsoft:

$>$ MS Visual Studio, version 10

$>$ NET framework, version 4.

$>$ MS SQL Server 2008.

The Online assistant to the module TC5 will consist in:

- Creation of connection (link) to the WEB-based system WebOptim and of other links to the Set of useful INTERNET resources;

- Development of e-content - Guidelines for work, Illustrative projects, Training modules for solving optimization problems in communications.

\subsection{Tasks to be solved by interaction between the modules TC2 and TC5}

Modern design in telecommunications is optimization oriented. Tasks for green communications consist in minimal power consumption, maximal efficiency, limited electromagnetic radiation to harmless levels, minimal thermal emission, minimal volume, weight and price, which are optimization single or multiple objective criterion tasks. Some optimization tasks in circuit design, as for example PROMETHEE - Based Approach for Multiple Objective Voltage Regulator Optimization are considered in [5]. Other optimization tasks in telecommunication system design are:

- Minimization of power consumption using reconfigurable FPGAs [7];

- Optimal numbers of sensors and antennas and minimal costs in smart cities projects [13];

- Optimal location and distribution of virtual machines (VM) on servers in a cloud for minimal power consumption of the cloud [9];

- Energy efficient small cell scenario in heterogeneous wireless networks [10];

- L-DAS (Large-scale distributed antenna system) energy efficiency maximization problem through power consumption modeling [11];

- Optimal number of antennas, active users and transmit power in MIMO (multi input multi output) system uniformly covering a given area with maximal energy efficiency [12].

These and other optimization tasks in communication circuit and system design will be solved in OPTIMEK by interaction between the modules TC2 and TC5.

\section{Methods and tools for software realization of OPTIMEK}

The platform OPTIMEK will be based on fast Ethernet connection and elastic storage, so different files would be stored and accessible. The cloud accessibility concept of OPTIMEK is presented on Fig.4.

The main idea of the web portal is to be accessible from every device that has internet connection and to store different data and knowledge, as well as connections to selected free CAD systems for design in telecommunications. The portal will be deployed using HTML \& CSS for front end. For back end realization will be used PHP and MySQL, since the databases will be double secured. The design will be innovative, easy-to-use with intuitive methods for navigation through the platform. It will be available twenty four hours per day and seven days per week. The accent will be open source and free tools.

The code of OPTIMEK will be developed using the latest architectural pattern for implementing user interfaces - MVC (Model, View, Controller) [16]. So the software will be divided to three different parts which are interconnected. This model of programming and developing divide the web portal into three components: View, Controller and Model. 


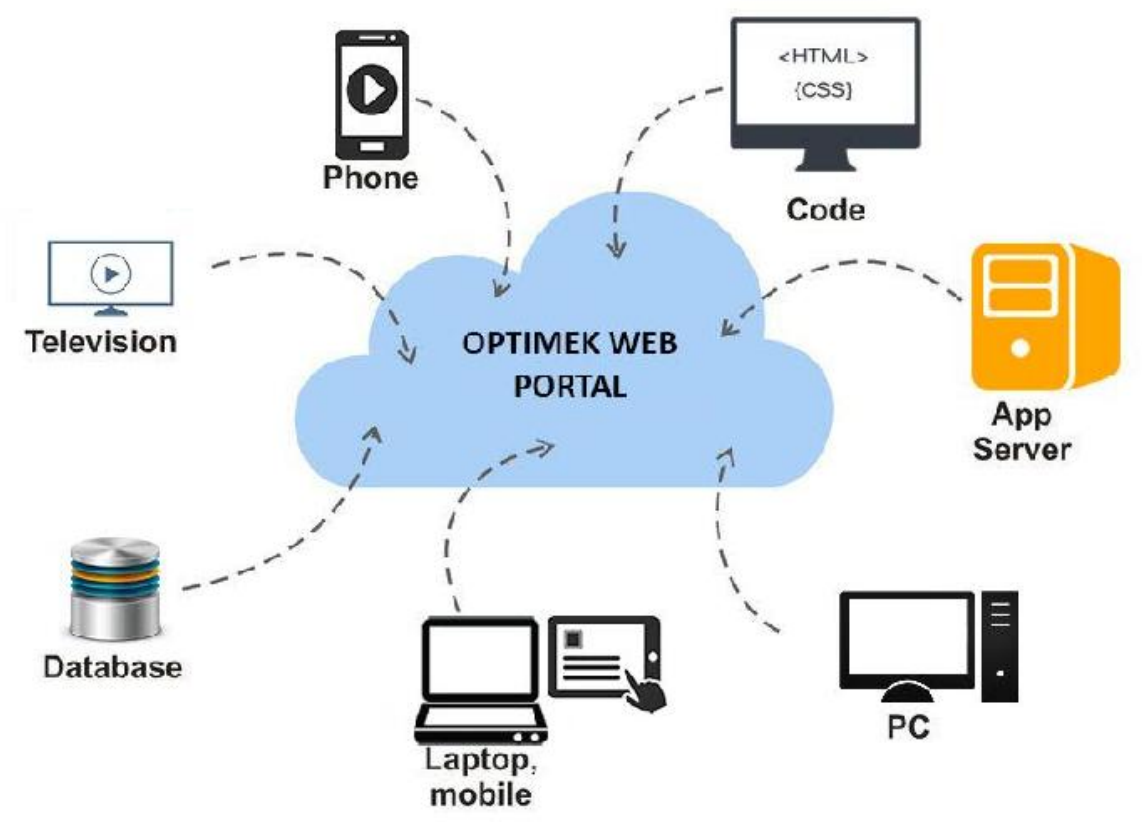

Fig.4. Cloud accessibility concept of OPTIMEK

The Controller is used to send commands to the model to update the model's state. It can also send commands to its associated view to change the view's presentation of the model. The Model notifies its associated view and controller when there has been a change in its state. This notification allows the views to produce updated output and the controller to change the available commands. The View requests information from the model that it uses to generate an output view and to represent it to the users. The Model takes place at the server side of the platform. At the client side sole the web browser works. The platform will be managed behind by a second web-based platform. It will work as a background to the website that the users will see. Sole the administrator and people who will update the content will have access to this back end software. The background system will take care for any content in the website. This will be the method for updating the content and materials on it.

\section{Illustration of tools in the Module TC2 and Cloud applications}

On Fig.5 is presented a Portal for online calculators for telecommunications. The portal integrates about 100 online tools for design of resistors, capacitors, inductors, transformers, heat sinks, crystal resonators, LED arrays, PCBs, SMPS, Smith Charts transmission line load matching, load speakers, microphones, antennas, timer 555 circuits, SDR open source platforms. The Portal is described in [3] and it can be found on the web address: http://mircheva.free.bg/index.html

A new online circuit calculator called SmartCircuitCalc [4] is developed to help the design of equalizers, oscillators, crystal filters and timer 555 circuits. SmartCircuitCalc is presented on Fig. 6 and can be found on the web address: http://niki.heatdesign.net/. 


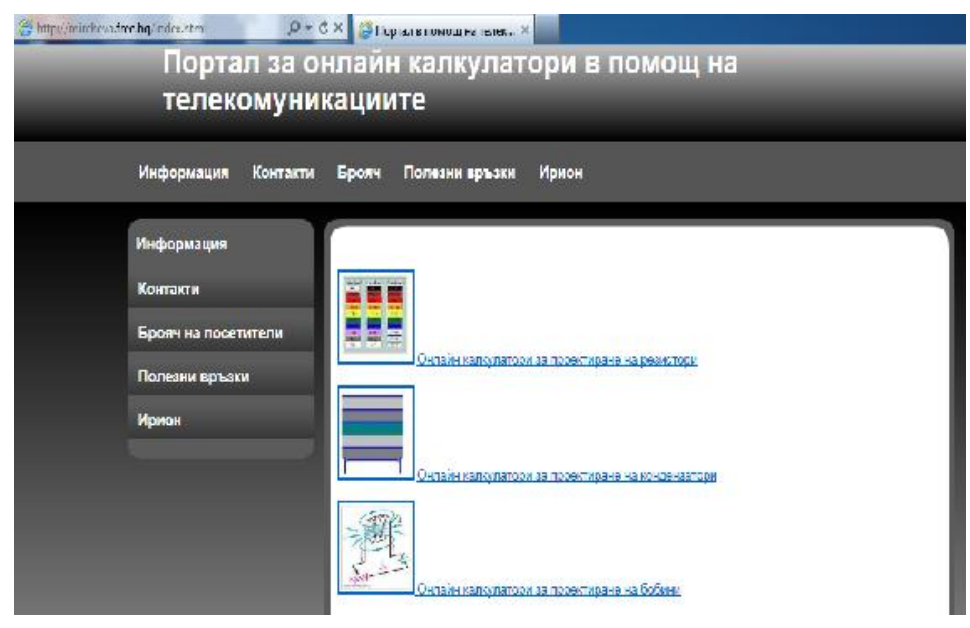

Fig. 5. Portal assisting web based CAD to be integrated in OPTIMEK

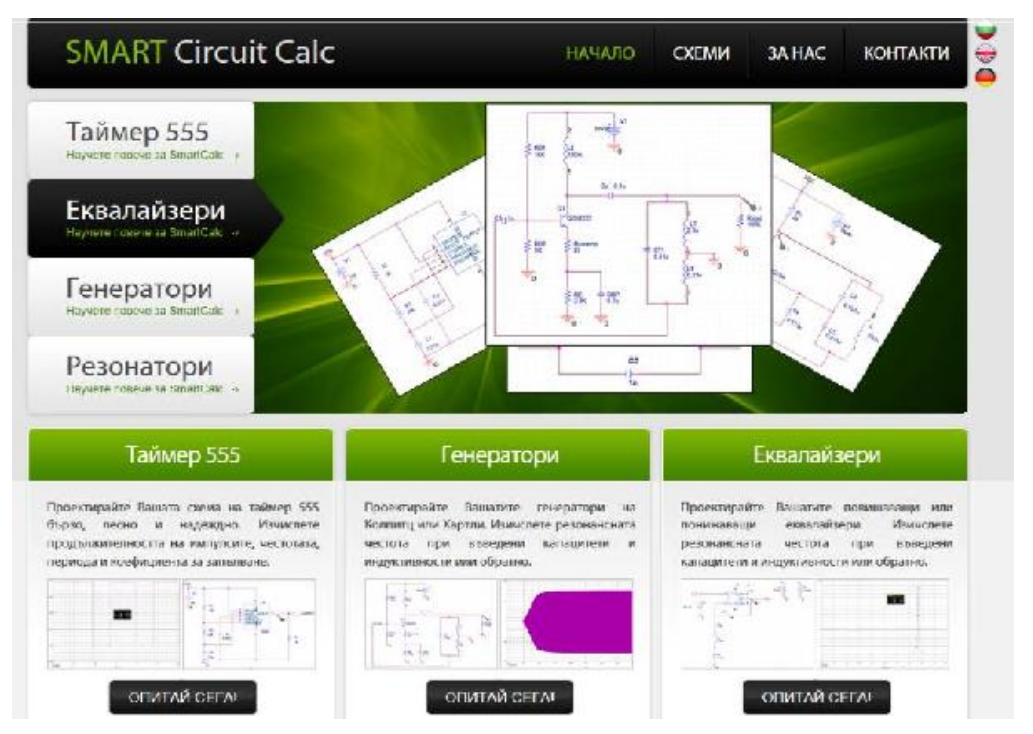

Fig. 6. Smart Circuit Calculator in OPTIMEK

SmartCircuitCalc is used to generate multiple projects for students in courses for Computer-aided design in telecommunications and it's integrated in a Moodle platform e-learning. SmartCircuitCalc will be integrated in the platform OPTIMEK and its concept will be considerably enlarged. 


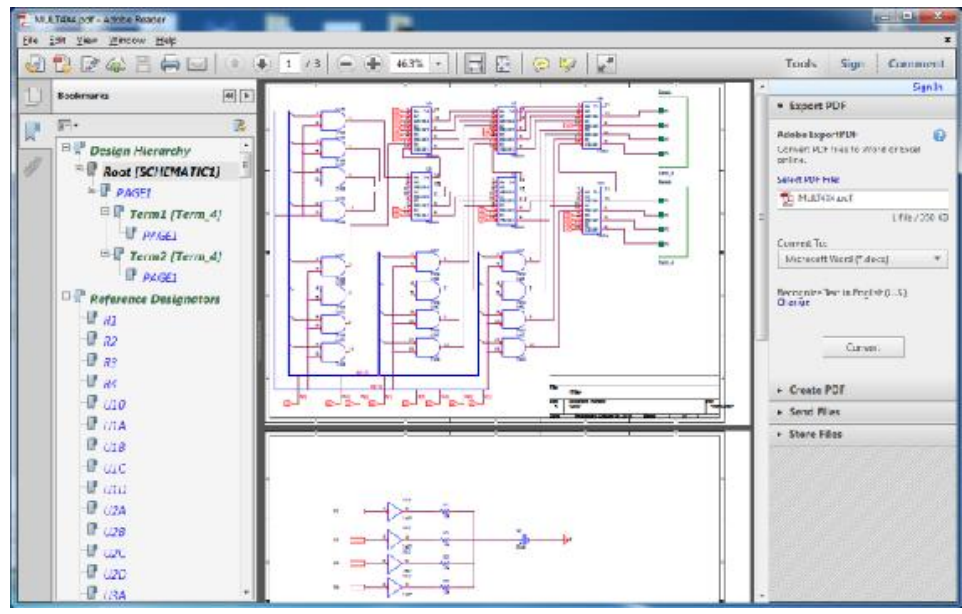

Fig.7. Intelligent .pdf file for hierarchical design of a multiplier $4 \times 4$ created in Cadence ORCAD 16.6

An illustration of cloud technologies application in existing CAD tools is given for the Cloud of Cadence tool ORCAD Design Suit 16.6. The ORCAD cloud uses Windows Azure Cloud Connector and permits to exchange ORCAD Capture projects saved as intelligent .pdf files [8]. On Fig.7 is presented an intelligent .pdf file generated for 2 levels hierarchical project of a 4-bit multiplier designed in ORCAD Capture. The ORCAD Cloud is free for ORCAD Capture projects but the tool nSWare PDF Generator for PCBs for sharing documentation for PCB designs in Allegro is a paid tool. The concept of cloudbased project exchange will also be used in the platform OPTIMEK to allow easy data and knowledge exchange.

\section{Conclusion}

The concept of the platform OPTIMEK described in the paper permits the integration of multiple online resources, which combined with available local resources will considerably help the design and management of communication systems. The software approach, methods and tools to be applied for the realization of OPTIMEK are described in details and tools, platforms and cloud technologies to be integrated in it are indicated and illustrated. A set of optimization tasks, which solution would be facilitated in OPTIMEK is defined.

\section{References}

1. G. Marinova, PSpice as a Verification Tool for Switch-Mode Power Supply Design with PowerEsim, $20^{\text {th }}$ IEEE workshop Nonlinear Dynamics of Electronic Systems - NDES 2012, 11.07.12 - 13.07.12, Wolfenbuttel, Germany, pp. 140-143, ISBN: 978-3-8007-3444-3

2. G. Marinova, Multitool Online Assisted Design of Communication Circuits and Systems, Proceedings of XLVII International scientific conference information, communication and energy systems and technologies, ICEST'2012, Veliko Tarnovo, Bulgaria, 28-30 June 2012, pp. 267-270, ISBN: 978-619-167-002-4

3. G. Marinova, Web assisted design of communication circuits and systems, Proceedings of the First International Conference on Telecommunications and remote sensing, ICTRS'2012, Sofia, Bulgaria, 29-30 August 2012, pp. 175-184, ISBN:978-989-8565-28-0

4. G.Marinova, N.Stoyanov, E-learning module for Computer-Aided Design im Moodle Platform, Proc. Of papers ICEST'2014, Serbia, Nis, June 25-27, 2014, Volume 2, pp.303-306, ISBN 978-86-6125$109-2$ 
5. G.Marinova, V. Guliashki, A PROMETHEE- Based Approach for Multiple Objective Voltage Regulator Optimization, Nonlinear Dynamics of Electronic Systems, Proc. Of $22^{\text {nd }}$ International Conference, NDES 2014, Albena, Bulgaria, July 4-6,2014, Springer, pp. 100-113, ISBN 978-3-31908671-2

6. G. Marinova, Z. Tchobanova, Simulation, Measurement and Test Environment for Pseudo Random Number Generator Circuits, Proceedings of the 20th IMEKO TC4 International Symposium "Research on Electrical and Electronic Measurement for the Economic Upturn" and 18th IMEKO TC-4 International Workshop on ADC and DAC Modelling and Testing, pp.. 833-838, ISBN 97892-990073-2-7

7. J. Palicot, H. Zhang, Cognitive Radio for Green Communications and networking, The Ninth Advanced International Conference on Telecommunications AICT 2013, June 23 - 28, 2013 - Rome, Italy

8. M. Waller, P. Willems, PDF Export for Capture Designs, Flow CAD Application Note PDF Export for Capture Designs, 9.9.2013

9. Ch. Morin, Snooze: A Scalable Energy-Efficient IaaS Cloud Management, System, Information Brochure NEXT-GWin'2014, Rennes, France, October 1-3, 2014, pp. 24

10. H. Bogucka, Energy-Efficient Small Cells Deployment in TV white Spaces, Information Brochure NEXT-GWin'2014, Rennes, France, October 1-3, 2014, pp. 27-28

11. S. Sun, An Energy-Efficient Large-Scale Distributed Antenna System for Multiple Users, Information Brochure NEXT-GWin'2014, Rennes, France, October 1-3, 2014, pp. 32-33

12. M.Debbah, Optimal Design of Energy-Efficient Multi-User MIMO Systems: Is Massive MIMO the Answer?, Information Brochure NEXT-GWin'2014, Rennes, France, October 1-3, 2014 , pp. 34-35

13. A. Hayar, Smart Cities: ICT R\&D Challenges and Opportunities, Information Brochure NEXTGWin'2014, Rennes, France, October 1-3, 2014, pp. 22-23

14. Kirilov L., Guliashki V., Genova K., Vassileva M., Staykov B., (2013) "Generalized scalarizing model GENS in DSS WebOptim”, International Journal of Decision Support System Technology, vol. 5, issue 3, pp. 1-11, Special Issue from the Decision Support Systems Stream on the EUROXXV Conference in Vilnius, (Sessions WA-27; WB-27; WC-27; WD-27). Guest Editors: Fatima Dargam, Shaofeng Liu, Isabelle Linden, DOI: 10.4018/IJDSST, ISSN: 1941-6296, EISSN: 1941-630X http://www.igi-global.com/journal/international-journal-decision-support-system/1120

15. Guliashki V., Genova K., Kirilov L., (2013) "The Decision Support System WebOptim in an ELearning Context", In: Proceedings of Papers of the International Conference "Automatics and Informatics'2013", 03.-07. October 2013, Sofia, Bulgaria, ISSN: 1313-1850, CD-ISSN: 1313-1869, pp. I-117 - I-120.

16. S. Masover, Model-View-Controller: A Design Pattern for Software, Berkley, June 2004 https://ist.berkeley.edu/as-ag/pub/pdf/mvc-seminar.pdf 